\title{
Heterosis for Grain Yield and its Component Traits in Rabi Sorghum
}

\author{
A. S. Totre ${ }^{1 *}$, A. S. Jadhav ${ }^{1}$, M. S. Shinde ${ }^{2}$, N. S. Kute ${ }^{3}$, U. S. Dalvi ${ }^{2}$, \\ R. S. Bhoge $e^{4}$ and G. C. Shinde ${ }^{1}$ \\ ${ }^{1}$ Department of Agricultural Botany, ${ }^{2}$ Sorghum Improvement Project, ${ }^{3}$ Pulses Improvement, \\ ${ }^{4}$ Seed Testing Research Unit, Seed Cell, Project MPKV, Rahuri, (MS), India \\ *Corresponding author
}

\section{A B S T R A C T}

\begin{tabular}{|l|}
\hline Key w or d s \\
Heterosis, L x T, \\
Rabi Sorghum \\
\hline Article Info \\
$\begin{array}{l}\text { Accepted: } \\
\text { 07 October } 2020 \\
\text { Available Online: } \\
\text { 10 November } 2020\end{array}$ \\
\hline
\end{tabular}

The experiment was undertaken on Line $\mathrm{x}$ Tester analysis for, grain yield and its component traits in crosses of $\mathrm{A}$ and $\mathrm{R}$ lines of rabi sorghum at All India Co-ordinated Sorghum Improvement Project, M.P.K.V., Rahuri, during the year 2017-18 with objectives to study the heterosis of parents and hybrids. The four CMS lines (females), ten testers (males) and their forty $\mathrm{F}_{1}$ 's hybrids were studied by using $\mathrm{L}$ x $\mathrm{T}$ design. Observations were recorded on eleven characters viz., Days to $50 \%$ flowering, Days to physiological maturity, seedling height at 14 DAE $(\mathrm{cm})$, Plant height $(\mathrm{cm})$, Grains per panicle (no.), Panicle weight (g), 1000 grain weight (g), Dry fodder weight (g), Dry matter content (g), Grain yield per plant (g) and Harvest index (\%). Among the eleven characters studied, majority of the characters exhibited mid-parents as well as better parent heterosis along with standard heterosis in desirable direction in most of the hybrids, indicating the predominant role of non-fixable inter-allelic interactions and over dominance in the expression of heterosis in respect of all these traits. Among the hybrids RMS-2010-16A $x$ RSV-2124, RMS-2010-24A x RSV-2124 and CMS-185A x RSV-2124, are identified as promising crosses.

\section{Introduction}

Sorghum [Sorghum bicolor (L.) Moench] is cultivated as a major food crop in several countries in South Asia, Africa and Central America. It is fifth important cereal of the world after wheat, maize, rice and barley (Dillon et al., 2007). Sorghum is believed to be originated in Africa and spread all over the world. Sorghum [Sorghum bicolor (L.) Moench] known as great millet and guinea corn in west Africa, kafir corn in South Africa, dura in Sudan, mtama in Eastern
Africa, jowar in India and kaoliang in China (Purseglove, 1972).

Sorghum (Sorghum bicolor (L.) Moench) is unique in its adaptation to extreme environmental conditions. In India, there are two distinct growing season for sorghum i.e. rainy (kharif) and post rainy (rabi) seasons. Rabi sorghum is highly valued for consumption purpose due to the excellent quality of the grain, which matures during rain free cool climate. Hence, this grain fetches high market price, almost double that of kharif grain. 
The discovery of cytoplasmic male sterile lines in sorghum facilitates the production of hybrids. Heterosis has been commercially exploited due to the availability of a stable and heritable CMS system to improve its productivity. Hence, the aim of the study was to evaluate the parents' genetic diversity and estimate percentage of heterosis of their $F_{1}$. Knowledge on the magnitude of heterosis for various characters is essential to locate better combinations to exploit them through heterosis breeding. Over dominance is attributed towards heterobeltiosis, while commercial superiority of the hybrid may be assessed by evaluating with a standard commercial check (Prakash et al., 2010). With this point of views the hybrids generated in the present investigation were evaluated and selected on the basis of their standard heterosis.

\section{Materials and Methods}

The experimental material for the present study comprised of four male sterile lines, ten restorers, their resulting forty hybrids and one hybrid check CSH-15R. During rabi 2017- 18 four male sterile lines and ten restores were sown at Sorghum Improvement Project, M.P.K.V., Rahuri and these lines and testers were crossed in Line $x$ Tester design to produce forty possible hybrids. The experiment was conducted during rabi 2018 by using fourteen parents, their forty hybrids along with one standard check CSH-15R at Sorghum Improvement Project, M.P.K.V., Rahuri. The observations were recorded on eleven characters viz., Days to $50 \%$ flowering, Days to physiological maturity, Seedling height at 14 DAE $(\mathrm{cm})$, Plant height (cm), Grains per panicle (no.), Panicle weight (g), 1000 grain weight (g), Dry fodder weight (g), Dry matter content (g), Grain yield per plant (g) and Harvest Index (\%). In the present investigation heterosis has been estimated over mid parent (Average / Relative heterosis) and better parent (heterobeltiosis) as per Fonesca and Patterson (1968).

\section{Results and Discussion}

The hybrids performed significantly better than the respective parents. Significant heterosis was observed for most of the studied characters. Average heterosis and heterobeltiosis in hybrids varied significantly and could be due to genetic diversity of parents used to generate the hybrids. This indicated the existence of vigor and development of hybrids. The analysis of variance and estimates of gca and sca variance are presented in Table 1 and 2 . It was observed that, the mean squares due to lines, testers as well as lines vs tester interaction and hybrids were found significant for all the characters under studies except line, tester and line vs tester in days to $50 \%$ flowering. This suggested that the experimental material possessed considerable amount of variability for grain yield and all the component traits. Mean performance of parents, hybrids and standard check for grain yield per plant and its contributing characters in rabi sorghum are presented in Table 3. Higher values are desirable for all traits under study except for days to $50 \%$ flowering and days to maturity for which lower values are preferred.

The mean performance of hybrids for different traits studied were compared with the corresponding mid parent (MP), better parent (BP) and standard check hybrids (CSH-15R) and the differences are being expressed as per cent heterosis for grain yield, its components traits. In rabi sorghum, positive heterosis was desirable for all the characters studied except days to $50 \%$ flowering and days to maturity where negative heterosis is desirable. Character wise results of average heterosis $\left(\mathrm{H}_{1}\right)$ heterobeltiosis $\left(\mathrm{H}_{2}\right)$ and standard heterosis $\left(\mathrm{H}_{3}\right)$ observed in the forty crosses (Table 4$)$. 
Table.1 Analysis of variance for combining ability in rabi sorghum

\begin{tabular}{|c|c|c|c|c|c|c|c|c|c|c|c|c|}
\hline Sources & DF & $\begin{array}{c}\text { Days to } \\
50 \% \\
\text { flowering }\end{array}$ & $\begin{array}{c}\text { Days to } \\
\text { physiologica } \\
\text { I maturity }\end{array}$ & $\begin{array}{c}\text { Seedling } \\
\text { height at } \\
14 \text { DAE } \\
\text { (cm) }\end{array}$ & $\begin{array}{l}\text { Plant height } \\
\text { (cm) }\end{array}$ & $\begin{array}{c}\text { Grains per } \\
\text { panicle (no.) }\end{array}$ & $\begin{array}{c}\text { Panicle } \\
\text { weight (g) }\end{array}$ & $\begin{array}{l}1000 \\
\text { grain } \\
\text { weight } \\
\text { (g) }\end{array}$ & $\begin{array}{c}\text { Dry } \\
\text { fodder } \\
\text { weight }(\mathrm{g})\end{array}$ & $\begin{array}{l}\text { Dry matter } \\
\text { content }(\mathrm{g})\end{array}$ & $\begin{array}{c}\text { Grain } \\
\text { yield per } \\
\text { plant (g) }\end{array}$ & $\begin{array}{c}\text { Harvest } \\
\text { index } \\
(\%)\end{array}$ \\
\hline Replications & 2 & 1.07 & $47.22 * *$ & $8.58 * *$ & 265.62 & $129953.71 * *$ & 196.30 & $45.90 * *$ & 30.27 & $2725.37 * *$ & 33.81 & $17.34 *$ \\
\hline Treatments & 53 & $13.40 * *$ & $22.07 * *$ & $8.80 * *$ & $1546.37 * *$ & $132943.91 * *$ & $559.93 * *$ & $41.66 * *$ & $456.61 * *$ & $1319.82 * *$ & $155.51 * *$ & $17.34 * *$ \\
\hline Parents & 13 & 3.03 & $16.76^{* *}$ & 1.30 & $3540.38 * *$ & $160844.19 * *$ & $675.45^{* *}$ & $71.50 * *$ & $475.64 * *$ & $1932.65 * *$ & $236.12 * *$ & $12.87 * *$ \\
\hline Line & 3 & 5.66 & $21.12 *$ & 0.74 & $686.84 * *$ & 48218.65 & $2206.62 * *$ & 4.30 & 58.43 & 249.98 & 62.76 & $24.06^{* *}$ \\
\hline Testers & 9 & 2.08 & $15.62 * *$ & 1.61 & $606.72 * *$ & $139852.04 * *$ & 228.99 & $58.03 * *$ & 99.35 & $1323.37 * *$ & $167.11 * *$ & 9.24 \\
\hline $\begin{array}{l}\text { Line } \quad \text { vs. } \\
\text { Tester }\end{array}$ & 1 & 3.65 & 14.00 & 0.16 & $38503.94 * *$ & $687650.07 * *$ & 100.15 & $394.40 * *$ & $5113.96 * *$ & $12464.15^{* *}$ & $1377.26 * *$ & 12.02 \\
\hline $\begin{array}{l}\text { Parent vs. } \\
\text { hybrid }\end{array}$ & 1 & $80.55 * *$ & $115.01 * *$ & $82.64 * *$ & $11360.11 * *$ & $999520.58 * *$ & 278.30 & $29.35 * *$ & $2790.35 * *$ & $12405.80 * *$ & $186.77 *$ & $86.63 * *$ \\
\hline Hybrids & 39 & $15.14 * *$ & $21.45 * *$ & $9.41 * *$ & $630.07 * *$ & $101423.90 * *$ & $528.64 * *$ & $32.03 * *$ & $390.43 * *$ & $831.28 * *$ & $127.84 * *$ & $17.06^{* *}$ \\
\hline Error & 106 & 2.40 & 5.80 & 0.93 & 168.89 & 19771.94 & 167.83 & 2.81 & 92.52 & 237.04 & 31.25 & 4.92 \\
\hline
\end{tabular}

Note: * Significant at $5 \%$ level of significance, ** Significant at $1 \%$ level of significance

Table.2 Estimates of combining ability in rabi sorghum

\begin{tabular}{|c|c|c|c|c|c|c|c|c|c|c|c|}
\hline Estimates & $\begin{array}{c}\text { Days to } \\
50 \% \\
\text { flowering }\end{array}$ & $\begin{array}{c}\text { Days to } \\
\text { physiological } \\
\text { maturity }\end{array}$ & $\begin{array}{l}\text { Seedling } \\
\text { height at } \\
14 \text { DAE } \\
\text { (cm) }\end{array}$ & $\begin{array}{c}\text { Plant } \\
\text { height }(\mathbf{c m})\end{array}$ & $\begin{array}{c}\text { Grains per } \\
\text { panicle (no.) }\end{array}$ & $\begin{array}{l}\text { Panicle } \\
\text { weight } \\
\text { (g) }\end{array}$ & $\begin{array}{c}1000 \\
\text { grain } \\
\text { weight } \\
\text { (g) }\end{array}$ & $\begin{array}{c}\text { Dry } \\
\text { fodder } \\
\text { weight (g) }\end{array}$ & $\begin{array}{l}\text { Dry matter } \\
\text { content (g) }\end{array}$ & $\begin{array}{l}\text { Grain } \\
\text { yield per } \\
\text { plant }(g)\end{array}$ & $\begin{array}{c}\text { Harvest } \\
\text { index } \\
(\%)\end{array}$ \\
\hline$\sigma_{\text {gca }}^{2}$ & $1.8042 * *$ & $2.1592 * *$ & $0.6555^{*}$ & $105.4407 * *$ & 3606.7121 & 8.2268 & 3.1135 & $41.9674 * *$ & 25.7846 & 3.1187 & $0.8453 *$ \\
\hline$\sigma_{\text {sca }}^{2}$ & $2.4902 * *$ & $2.7510 * *$ & $2.8027 * *$ & 10.4999 & $23175.9934 * *$ & $127.39 * *$ & 5.0730 & $65.4107 * *$ & $195.0548 * *$ & $\begin{array}{c}32.2124 * \\
*\end{array}$ & $3.4655^{* *} *$ \\
\hline$\sigma_{A}^{2}$ & 3.6084 & 4.3185 & 1.3109 & 210.88 & 7213.4242 & 16.4536 & 6.2270 & 83.9348 & 79.0161 & 6.2375 & 1.6906 \\
\hline$\sigma_{D}^{2}$ & 2.4902 & 2.7510 & 2.8027 & 10.4999 & 23175.9934 & 127.3987 & 5.0730 & 65.4107 & 195.0548 & 32.2124 & 3.4655 \\
\hline$\sigma_{A}^{2} / \sigma_{D}^{2}$ & 1.4490 & 1.5698 & 0.4677 & 20.08 & 0.3112 & 0.1292 & 1.2275 & 1.2832 & 0.2644 & 0.1936 & 0.4878 \\
\hline
\end{tabular}


Table.3 Mean performance of parents and hybrids for grain yield and its contributing characters in rabi sorghum

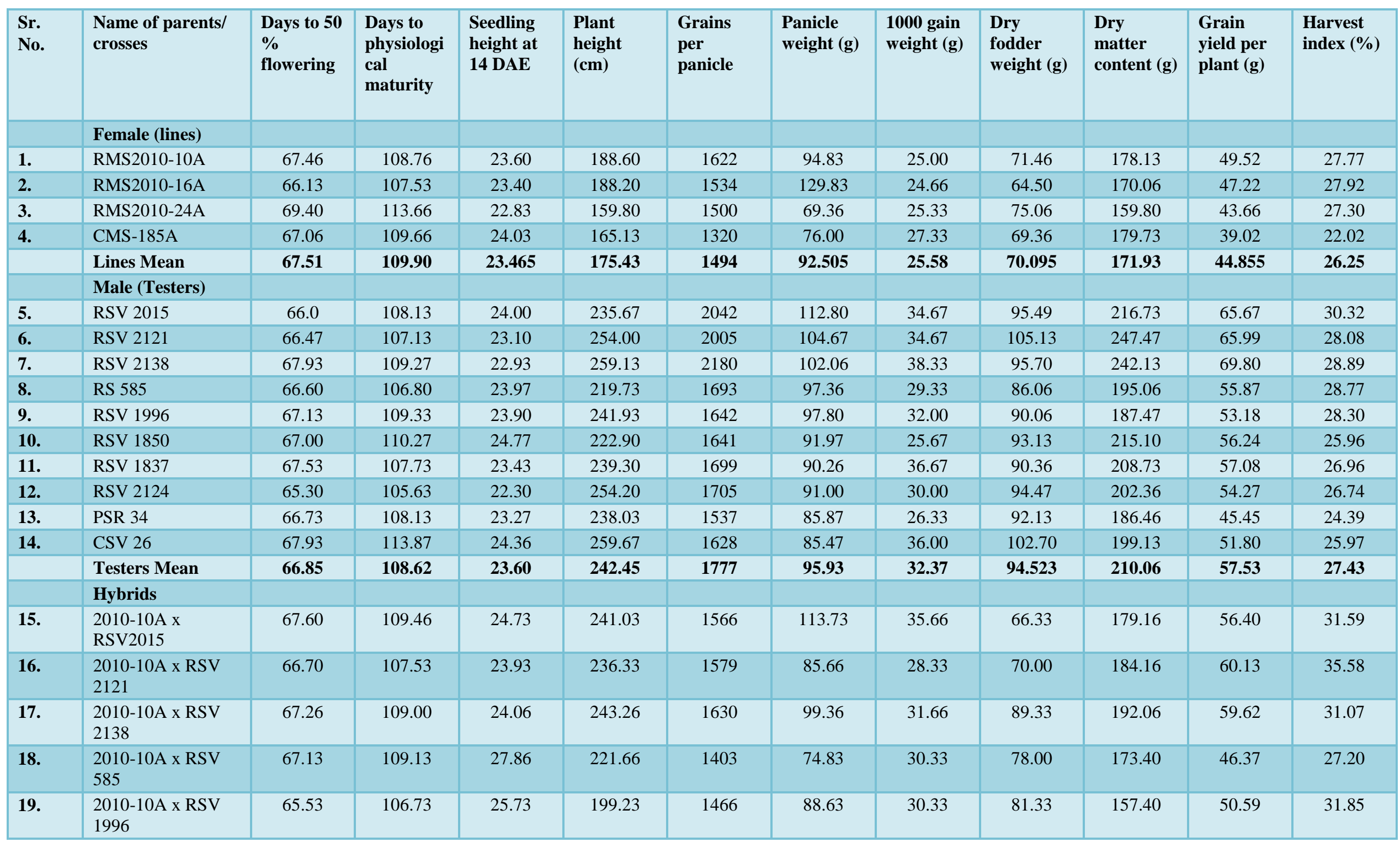


Int.J.Curr.Microbiol.App.Sci (2020) 9(11): 846-863

\begin{tabular}{|c|c|c|c|c|c|c|c|c|c|c|c|c|}
\hline 20. & $\begin{array}{l}2010-10 \mathrm{~A} \times \mathrm{RSV} \\
1850\end{array}$ & 62.00 & 104.33 & 30.66 & 208.20 & 1445 & 88.66 & 31.33 & 70.33 & 168.13 & 48.36 & 28.62 \\
\hline 21. & $\begin{array}{l}2010-10 \text { A x RSV } \\
1837\end{array}$ & 66.10 & 108.46 & 26.06 & 218.40 & 1624 & 90.80 & 38.33 & 93.53 & 196.80 & 57.65 & 29.69 \\
\hline 22. & $\begin{array}{l}2010-10 \text { A x RSV } \\
2124\end{array}$ & 66.00 & 107.40 & 25.60 & 211.13 & 1231 & 83.73 & 28.33 & 64.60 & 162.40 & 40.51 & 25.30 \\
\hline 23. & 2010-10A x PSR 34 & 65.86 & 106.93 & 25.93 & 214.26 & 1504 & 104.00 & 29.33 & 82.60 & 167.06 & 50.52 & 30.65 \\
\hline 24. & $\begin{array}{l}2010-10 \mathrm{~A} \times \mathrm{CSV} \\
26\end{array}$ & 69.80 & 111.20 & 28.26 & 215.93 & 1511 & 109.80 & 32.33 & 82.80 & 199.46 & 58.54 & 29.20 \\
\hline 25. & $\begin{array}{l}2010-16 \text { A x RSV } \\
2015\end{array}$ & 62.90 & 103.30 & 26.96 & 207.06 & 1473 & 81.93 & 36.00 & 86.13 & 168.70 & 43.24 & 25.68 \\
\hline 26. & $\begin{array}{l}2010-16 \text { A x RSV } \\
2121\end{array}$ & 64.13 & 105.13 & 26.50 & 197.76 & 1549 & 98.00 & 29.33 & 89.80 & 176.40 & 49.12 & 27.78 \\
\hline 27. & $\begin{array}{l}2010-16 \mathrm{~A} \times \mathrm{RSV} \\
2015\end{array}$ & 67.40 & 108.66 & 23.70 & 204.60 & 1616 & 96.23 & 32.33 & 77.80 & 167.13 & 51.63 & 29.59 \\
\hline 28. & $\begin{array}{l}2010-16 \text { A x RSV } \\
2138\end{array}$ & 67.40 & 108.46 & 23.90 & 197.00 & 1376 & 76.73 & 28.00 & 80.86 & 183.06 & 49.83 & 28.29 \\
\hline 29. & 2010-16A x RS 585 & 65.60 & 107.40 & 22.93 & 192.73 & 1394 & 75.73 & 29.00 & 86.06 & 164.73 & 45.41 & 27.72 \\
\hline 30. & $\begin{array}{l}2010-16 \text { A x RSV } \\
1996\end{array}$ & 67.66 & 109.53 & 25.46 & 188.96 & 1515 & 89.13 & 37.66 & 70.83 & 161.66 & 49.36 & 30.60 \\
\hline 31. & $\begin{array}{l}2010-16 \text { A x RSV } \\
1837\end{array}$ & 67.33 & 109.53 & 28.20 & 210.66 & 1638 & 105.40 & 32.66 & 83.13 & 190.13 & 55.48 & 29.33 \\
\hline 32. & $\begin{array}{l}2010-16 \text { A x RSV } \\
2124\end{array}$ & 66.80 & 109.00 & 23.20 & 204.46 & 2150 & 125.30 & 34.33 & 97.80 & 236.13 & 70.48 & 30.33 \\
\hline 33. & 2010-16A x PSR 34 & 62.60 & 105.53 & 25.53 & 210.33 & 1376 & 87.46 & 30.66 & 91.80 & 190.40 & 50.20 & 26.53 \\
\hline 34. & $\begin{array}{l}2010-16 \mathrm{~A} \times \mathrm{CSV} \\
26\end{array}$ & 65.66 & 106.33 & 27.03 & 209.86 & 1518 & 83.50 & 29.66 & 120.46 & 213.13 & 50.69 & 23.74 \\
\hline 35. & $\begin{array}{l}2010-24 \text { A x RSV } \\
2015\end{array}$ & 64.26 & 104.40 & 26.36 & 191.33 & 1882 & 111.83 & 35.00 & 73.66 & 183.83 & 60.63 & 32.82 \\
\hline 36. & $\begin{array}{l}2010-24 \mathrm{~A} \times \mathrm{RSV} \\
2121\end{array}$ & 64.13 & 105.13 & 24.73 & 189.46 & 1337 & 76.66 & 27.00 & 71.80 & 178.16 & 47.01 & 26.40 \\
\hline 37. & $\begin{array}{l}2010-24 \mathrm{~A} \times \mathrm{RSV} \\
2138\end{array}$ & 64.60 & 105.73 & 25.76 & 204.83 & 1532 & 89.40 & 30.00 & 70.06 & 190.66 & 56.06 & 29.68 \\
\hline 38. & 2010-24A x RS 585 & 66.20 & 107.53 & 24.56 & 192.56 & 1619 & 89.83 & 28.33 & 77.80 & 188.13 & 54.48 & 28.85 \\
\hline 39. & $\begin{array}{l}2010-24 \text { A x RSV } \\
1996\end{array}$ & 68.86 & 113.13 & 26.03 & 187.80 & 1341 & 103.73 & 26.00 & 60.46 & 162.80 & 45.34 & 28.00 \\
\hline 40. & $\begin{array}{l}2010-24 \mathrm{~A} \times \mathrm{RSV} \\
1850\end{array}$ & 69.13 & 112.63 & 24.10 & 189.83 & 1465 & 77.40 & 30.33 & 61.40 & 154.73 & 46.52 & 29.66 \\
\hline
\end{tabular}




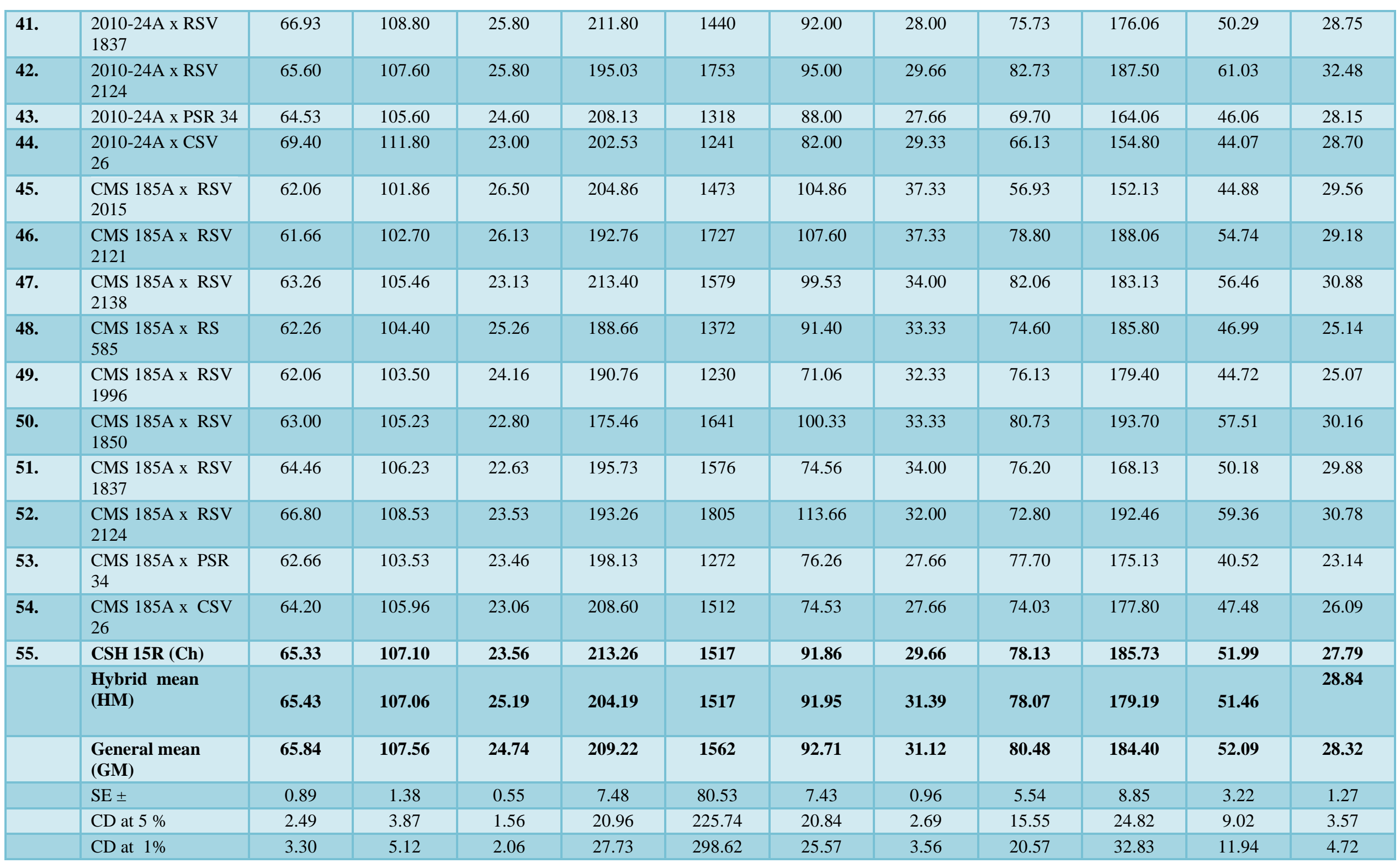


Table.4 Heterosis (\%) over mid-parent (MP), better-parent (BP) and standard check (CSH-15R) for different characters in rabi sorghum

\begin{tabular}{|c|c|c|c|c|c|c|c|c|c|c|}
\hline \multirow[t]{3}{*}{$\begin{array}{l}\text { Sr. } \\
\text { No. }\end{array}$} & \multirow[t]{3}{*}{ Crosses } & \multirow{2}{*}{\multicolumn{3}{|c|}{ Days to $50 \%$ flowering }} & \multicolumn{3}{|c|}{ Days to physiological maturity } & \multicolumn{3}{|c|}{$\begin{array}{c}\text { Seedling height at } 14 \text { DAE } \\
(\mathrm{cm})\end{array}$} \\
\hline & & & & & & 2 & & & 3 & \\
\hline & & $\mathrm{MP}(\mathrm{H} 1)$ & $\mathrm{BP}(\mathrm{H} 2)$ & $\begin{array}{l}\text { Check } \\
\text { (H3) }\end{array}$ & $\mathrm{MP}(\mathrm{H} 1)$ & $\mathrm{BP}(\mathrm{H} 2)$ & $\begin{array}{c}\text { Check } \\
\text { (H3) }\end{array}$ & $\mathrm{MP}(\mathrm{H} 1)$ & $\mathrm{BP}(\mathrm{H} 2)$ & $\begin{array}{c}\text { Check } \\
\text { (H3) }\end{array}$ \\
\hline 1. & 2010-10A x RSV 2015 & 1.30 & 2.42 & 3.74 & 0.94 & 1.23 & 2.21 & 3.92 & 3.06 & 4.95 \\
\hline 2. & 2010-10A x RSV 2121 & -0.40 & 0.35 & 2.09 & -0.39 & 0.37 & 0.40 & 2.50 & 1.41 & 1.56 \\
\hline 3. & 2010-10A x RSV 2138 & -0.64 & -0.30 & 2.96 & -0.02 & 0.21 & 1.77 & 3.44 & 1.98 & 2.12 \\
\hline 4. & 2010-10A x RSV 585 & 0.15 & 0.80 & 2.76 & 1.25 & 2.18 & 1.90 & $17.17 * *$ & $16.27 * *$ & $18.25^{* *}$ \\
\hline 5. & 2010-10A x RSV 1996 & -2.63 & -2.38 & 0.31 & -2.12 & -1.87 & -0.34 & $8.35 * *$ & $7.67 *$ & $9.19 * *$ \\
\hline 6. & 2010-10A x RSV 1850 & $-7.78 * *$ & $-7.46 * *$ & $-5.10 *$ & $-4.73 * *$ & $-4.08 *$ & -2.58 & $26.81 * *$ & $23.82 * *$ & $30.13 * *$ \\
\hline 7. & 2010-10A x RSV 1837 & -2.07 & -2.03 & 1.17 & 0.20 & 0.68 & 1.28 & $10.84 * *$ & $10.45 * *$ & $10.61 * *$ \\
\hline 8. & 2010-10A x RSV 2124 & -0.58 & 1.07 & 1.02 & 0.19 & 1.67 & 0.28 & $11.55^{* *}$ & $8.47 *$ & $8.63 *$ \\
\hline 9. & 2010-10A x PSR 34 & -1.84 & -1.30 & 0.82 & -1.40 & -1.11 & -0.16 & $10.67 * *$ & $9.89 * *$ & $10.04 * *$ \\
\hline 10. & 2010-10A x CSV 26 & 3.10 & 3.46 & $6.84 * *$ & -0.10 & 2.24 & $3.83 *$ & $17.86^{* *}$ & $16.01 * *$ & $19.94 * *$ \\
\hline 11. & 2010-16A x RSV 2015 & $-4.79 * *$ & $-4.70 *$ & -3.72 & $-4.20 * *$ & $-3.94 *$ & -3.55 & $13.78 * *$ & $12.36 * *$ & $14.43 * *$ \\
\hline 12. & 2010-16A x RSV 2121 & -3.27 & -3.02 & -1.84 & -2.05 & -1.87 & -1.84 & $13.98 * *$ & $13.25 * *$ & $12.45^{* *}$ \\
\hline 13. & 2010-16A x RSV 2015 & 0.55 & 1.92 & 3.16 & 0.25 & 1.05 & 1.46 & 2.30 & 1.28 & 0.57 \\
\hline 14. & 2010-16A x RSV 2138 & 1.56 & 1.92 & 3.16 & 1.21 & 1.56 & 1.28 & 0.91 & -0.28 & 1.41 \\
\hline 15. & 2010-16A x RS 585 & -1.55 & -0.81 & 0.41 & -0.95 & -0.12 & 0.28 & -3.03 & -4.04 & -2.69 \\
\hline 16. & 2010-16A x RSV 1996 & 1.65 & 2.32 & 3.57 & 0.58 & 1.86 & 2.27 & $5.74 * *$ & 2.83 & $8.06^{*}$ \\
\hline 17. & 2010-16A x RSV 1837 & 0.75 & 1.81 & 3.06 & 1.77 & 1.86 & 2.27 & $20.43 * *$ & $20.34 * *$ & $19.66^{* *}$ \\
\hline 18. & 2010-16A x RSV 2124 & 1.65 & 2.30 & 2.24 & 2.27 & 3.19 & 1.77 & 1.53 & -0.85 & -1.56 \\
\hline 19. & 2010-16A x PSR 34 & $-5.77 * *$ & $-5.34 * *$ & $-4.18 *$ & -2.13 & -1.86 & -1.46 & $9.43 * *$ & $9.12 * *$ & $8.35^{*}$ \\
\hline 20. & 2010-16A x CSV 26 & -2.04 & -0.71 & 0.51 & $-3.94 *$ & -1.12 & -0.72 & $13.19 * *$ & $10.94 * *$ & $14.71 * *$ \\
\hline
\end{tabular}

Note: * Significant at $5 \%$ level of significance, $* *$ Significant at $1 \%$ level of significance 
Contid...

\begin{tabular}{|c|c|c|c|c|c|c|c|c|c|c|}
\hline \multirow[t]{3}{*}{$\begin{array}{l}\text { Sr. } \\
\text { No. }\end{array}$} & \multirow[t]{3}{*}{ Crosses } & \multirow{2}{*}{\multicolumn{3}{|c|}{ Days to $50 \%$ flowering }} & \multirow{2}{*}{\multicolumn{3}{|c|}{ Days to physiological maturity }} & \multicolumn{3}{|c|}{$\begin{array}{c}\text { Seedling height at } 14 \text { DAE } \\
(\mathrm{cm})\end{array}$} \\
\hline & & & & & & & & & 3 & \\
\hline & & $\mathrm{MP}(\mathrm{H} 1)$ & $\mathrm{BP}(\mathrm{H} 2)$ & $\begin{array}{l}\text { Check } \\
\text { (H3) }\end{array}$ & $\mathrm{MP}(\mathrm{H} 1)$ & $\mathrm{BP}(\mathrm{H} 2)$ & $\begin{array}{l}\text { Check } \\
\text { (H3) }\end{array}$ & $\mathrm{MP}(\mathrm{H} 1)$ & $\mathrm{BP}(\mathrm{H} 2)$ & $\begin{array}{c}\text { Check } \\
\text { (H3) }\end{array}$ \\
\hline 21. & 2010-24A x RSV 2015 & $-5.07 * *$ & -2.63 & -1.63 & $-5.86 * *$ & -3.45 & -2.52 & $12.60 * *$ & $9.86^{* *}$ & $11.88 * *$ \\
\hline 22. & 2010-24A x RSV 2121 & $-5.59 * *$ & -3.51 & -1.84 & $-4.77 * *$ & -1.87 & -1.84 & $7.69 *$ & $7.07^{*}$ & 4.95 \\
\hline 23. & 2010-24A x RSV 2138 & $-5.92 * *$ & $-4.91 *$ & -1.12 & $-5.14 * *$ & -3.23 & -1.28 & $12.60 * *$ & $12.35 * *$ & $9.34 * *$ \\
\hline 24. & 2010-24A x RS 585 & -2.65 & -0.60 & 1.33 & -2.45 & 0.69 & 0.40 & 4.99 & 2.50 & 4.24 \\
\hline 25. & 2010-24A x RSV 1996 & 0.88 & 2.58 & $5.41 * *$ & 1.46 & 3.48 & $5.63 * *$ & $11.41 * *$ & $8.93 * *$ & $10.47 * *$ \\
\hline 26. & 2010-24A x RSV 1850 & 1.37 & 3.18 & $5.82 * *$ & 0.60 & 2.15 & $5.17 * *$ & 1.26 & -2.69 & 2.26 \\
\hline 27. & 2010-24A x RSV 1837 & -2.24 & -0.89 & 2.45 & -1.72 & 0.99 & 1.59 & $11.53 * *$ & $10.10 * *$ & $9.48 * *$ \\
\hline 28. & 2010-24A x RSV 2124 & -2.60 & 0.46 & 0.41 & -1.87 & 1.86 & 0.47 & $14.33 * *$ & $12.99 * *$ & $9.48 * *$ \\
\hline 29. & 2010-24A x PSR 34 & $-5.19 *$ & -3.30 & -1.22 & $-4.78 * *$ & -2.34 & -1.40 & 6.72 & 5.73 & 4.38 \\
\hline 30. & 2010-24A x CSV 26 & 1.07 & 2.16 & $6.22 * *$ & -1.73 & -1.64 & $4.39 *$ & -2.54 & -5.61 & -2.40 \\
\hline 31. & CMS 185A x RSV 2015 & $-6.71 * *$ & $-5.96 * *$ & $-5.00^{*}$ & $-6.46 * *$ & $-5.80 * *$ & $-4.89 * *$ & $10.34 * *$ & $10.26 * *$ & $12.45^{* *}$ \\
\hline 32. & CMS 185A x RSV 2121 & $-7.64 * *$ & $-7.22 * *$ & $-5.61 * *$ & $-5.26 * *$ & $-4.14 * *$ & $-4.11 *$ & $10.89 * *$ & $8.74 * *$ & $10.89 * *$ \\
\hline 33. & CMS $185 \mathrm{~A}$ x RSV 2138 & $-6.27 * *$ & $-5.67 * *$ & -3.16 & $-3.65^{*}$ & -3.48 & -1.53 & -1.49 & -3.74 & -1.84 \\
\hline 34. & CMS 185A x RS 585 & $-6.83 * *$ & $-6.51 * *$ & $-4.69 *$ & $-3.54 * *$ & -2.25 & -2.52 & 5.28 & 5.13 & $7.21 *$ \\
\hline 35. & CMS 185A x RSV 1996 & $-7.50 * *$ & $-7.46^{* *}$ & $-5.00 *$ & $-5.48 * *$ & $-5.34 * *$ & -3.36 & 0.83 & 0.55 & 2.55 \\
\hline 36. & CMS 185A x RSV 1850 & $-6.02 * *$ & $-5.97 * *$ & -3.57 & $-4.30 * *$ & $-4.04 *$ & -1.74 & $-6.56^{*}$ & $-7.94 *$ & -3.25 \\
\hline 37. & CMS 185A x RSV 1837 & $-4.21 *$ & $-3.88 *$ & -1.33 & -2.27 & -1.39 & -0.81 & -4.63 & -5.83 & -3.96 \\
\hline 38. & CMS 185A x RSV 2124 & 0.93 & 2.30 & 2.24 & 0.82 & 2.75 & 1.34 & 1.58 & -2.08 & -0.14 \\
\hline 39. & CMS 185A x PSR 34 & $-6.33 * *$ & $-6.09 * *$ & $-4.08 *$ & $-4.93 * *$ & $-4.25^{*}$ & -3.33 & -0.78 & -2.36 & -0.42 \\
\hline \multirow[t]{2}{*}{40} & CMS 185A x CSV 26 & $-4.89 * *$ & $-4.27 *$ & -1.73 & $-5.19 * *$ & -3.37 & -1.06 & -4.68 & -5.34 & -2.12 \\
\hline & S.E. $($ Sij $) \pm$ & 1.097 & 1.27 & 1.27 & 1.70 & 1.96 & 1.96 & 0.68 & 0.78 & 0.78 \\
\hline
\end{tabular}

Note: * Significant at $5 \%$ level of significance, ${ }^{* *}$ Significant at $1 \%$ level of significance 
Contid...

\begin{tabular}{|c|c|c|c|c|c|c|c|c|c|c|}
\hline \multirow[t]{3}{*}{ Sr. No. } & \multirow[t]{3}{*}{ Crosses } & \multicolumn{3}{|c|}{ Plant height $(\mathrm{cm})$} & \multicolumn{3}{|c|}{ Grains per panicles (no.) } & \multicolumn{3}{|c|}{ Panicle weight (g) } \\
\hline & & \multicolumn{3}{|c|}{4} & \multicolumn{3}{|c|}{5} & \multicolumn{3}{|c|}{6} \\
\hline & & $\mathrm{MP}(\mathrm{H} 1)$ & $\mathrm{BP}(\mathrm{H} 2)$ & $\begin{array}{l}\text { Check } \\
(\mathrm{H} 3)\end{array}$ & $\mathrm{MP}(\mathrm{H} 1)$ & $\mathrm{BP}(\mathrm{H} 2)$ & $\begin{array}{c}\text { Check } \\
\text { (H3) }\end{array}$ & $\mathrm{MP}(\mathrm{H} 1)$ & $\mathrm{BP}(\mathrm{H} 2)$ & $\begin{array}{c}\text { Check } \\
\text { (H3) }\end{array}$ \\
\hline 1. & 2010-10A x RSV 2015 & $13.62 * *$ & 2.28 & $13.02 *$ & $-14.51 * *$ & $-23.31 * *$ & 2.89 & 9.55 & 0.83 & $23.80 *$ \\
\hline 2. & 2010-10A x RSV 2121 & 6.79 & -6.96 & $10.82 *$ & $-12.93 *$ & $-21.26 * *$ & 3.72 & -14.12 & -18.15 & -6.75 \\
\hline 3. & 2010-10A x RSV 2138 & $8.67 *$ & -6.12 & $14.07 * *$ & $-14.25 * *$ & $-25.22 * *$ & 7.06 & 0.93 & -2.65 & 8.16 \\
\hline 4. & 2010-10A x RSV 585 & 8.57 & 0.88 & 3.94 & $-15.33^{*}$ & $-17.11 *$ & -7.83 & $-22.13^{* *}$ & $-23.14^{*}$ & -18.54 \\
\hline 5. & 2010-10A x RSV 1996 & -7.45 & $-17.65 * *$ & -6.58 & -10.11 & -10.66 & -3.66 & -7.98 & -9.37 & -3.52 \\
\hline 6. & 2010-10A x RSV 1850 & 1.19 & -6.59 & -2.38 & -11.42 & -11.95 & -5.06 & -5.07 & -6.50 & -3.48 \\
\hline 7. & 2010-10A x RSV 1837 & 2.08 & -8.73 & 2.41 & -2.16 & -4.38 & 6.70 & -1.89 & -4.25 & -1.16 \\
\hline 8. & 2010-10A x RSV 2124 & -4.64 & $-16.94 * *$ & -1.00 & $-25.99 * *$ & $-27.81 * *$ & $-19.12 *$ & -9.88 & -11.70 & -8.85 \\
\hline 9. & 2010-10A x PSR 34 & 0.45 & -9.98 & 0.47 & -4.79 & -7.27 & -1.22 & 15.11 & 9.67 & 13.21 \\
\hline 10. & 2010-10A x CSV 26 & -3.66 & $-16.84 * *$ & 1.25 & -6.97 & -7.15 & -0.71 & $21.80 *$ & 15.78 & 19.52 \\
\hline 11. & 2010-16A x RSV 2015 & -2.30 & $-12.14 * *$ & -2.91 & $-17.63 * *$ & $-27.87 * *$ & -3.23 & $-32.46 * *$ & $-36.89 * *$ & -10.81 \\
\hline 12. & 2010-16A x RSV 2121 & $-10.55^{*}$ & $-22.14 * *$ & -7.27 & $-12.49 *$ & $-22.76^{* *}$ & 1.73 & $-16.42 *$ & $-24.52 * *$ & 6.68 \\
\hline 13. & 2010-16A x RSV 2015 & $-8.52 *$ & $-21.04 * *$ & -4.06 & $-12.96 *$ & $-25.84 * *$ & 6.18 & $-17.00 *$ & $-25.88 * *$ & 4.75 \\
\hline 14. & 2010-16A x RSV 2138 & -3.42 & -10.35 & -7.63 & $-14.72 *$ & $-18.71 * *$ & -9.61 & $-32.45 * *$ & $-40.90 * *$ & -16.47 \\
\hline 15. & 2010-16A x RS 585 & $-10.38 *$ & $-20.34 * *$ & -9.63 & -12.18 & $-15.05^{*}$ & -8.39 & $-33.46^{* *}$ & $-41.67 * *$ & -17.56 \\
\hline 16. & 2010-16A x RSV 1996 & -8.07 & $-15.22 * *$ & $-11.39 *$ & -4.59 & -7.70 & -0.48 & $-19.63 *$ & $-31.35 * *$ & -2.98 \\
\hline 17. & 2010-16A x RSV 1837 & -1.44 & $-11.97 * *$ & -1.22 & 1.33 & -3.57 & 7.60 & -4.23 & $-18.82 *$ & 14.73 \\
\hline 18. & 2010-16A x RSV 2124 & -7.56 & $-19.56 * *$ & -4.13 & $32.71 * *$ & $26.05 * *$ & $41.22 * *$ & 13.48 & -3.49 & $36.39 * *$ \\
\hline 19. & 2010-16A x PSR 34 & -1.31 & $-11.64 * *$ & -1.38 & -10.39 & -10.47 & -9.59 & $-18.90 *$ & $-32.63 * *$ & -4.79 \\
\hline 20. & 2010-16A x CSV 26 & -6.28 & $-19.18 * *$ & -1.59 & -4.00 & -6.75 & -0.29 & $-22.43^{*}$ & $-35.69 * *$ & -9.11 \\
\hline
\end{tabular}

Note: * Significant at $5 \%$ level of significance, $* *$ Significant at $1 \%$ level of significance 
Contid...

\begin{tabular}{|c|c|c|c|c|c|c|c|c|c|c|}
\hline \multirow[t]{3}{*}{ Sr. No. } & \multirow[t]{3}{*}{ Crosses } & \multicolumn{3}{|c|}{ Plant height (cm) } & \multicolumn{3}{|c|}{ Grains per panicles } & \multicolumn{3}{|c|}{ Panicle weight (g) } \\
\hline & & \multicolumn{3}{|c|}{4} & \multicolumn{3}{|c|}{5} & \multicolumn{3}{|c|}{6} \\
\hline & & $\mathrm{MP}(\mathrm{H} 1)$ & $\mathrm{BP}(\mathrm{H} 2)$ & $\begin{array}{c}\text { Check } \\
\text { (H3) }\end{array}$ & $\mathrm{MP}(\mathrm{H} 1)$ & $\mathrm{BP}(\mathrm{H} 2)$ & $\begin{array}{c}\text { Check } \\
\text { (H3) }\end{array}$ & $\mathrm{MP}(\mathrm{H} 1)$ & $\mathrm{BP}(\mathrm{H} 2)$ & $\begin{array}{c}\text { Check } \\
\text { (H3) }\end{array}$ \\
\hline 21. & 2010-24A x RSV 2015 & -3.42 & $-18.81 * *$ & $-10.28 *$ & 6.26 & -7.85 & $23.64 * *$ & $22.78^{*}$ & -0.86 & 21.73 \\
\hline 22. & 2010-24A x RSV 2121 & -8.43 & $-25.41 * *$ & $-11.16^{*}$ & $-23.70 * *$ & $-33.31 * *$ & -12.15 & -11.89 & $-26.75 * *$ & -16.55 \\
\hline 23. & 2010-24A x RSV 2138 & -2.21 & $-20.95 * *$ & -3.95 & $-16.70 * *$ & $-29.69 * *$ & 0.67 & 4.30 & -12.41 & -2.69 \\
\hline 24. & $2010-24 \mathrm{~A} \times \mathrm{RS} 585$ & 1.48 & $-12.36 * *$ & -9.71 & 1.44 & -4.34 & 6.37 & 7.76 & -7.74 & -2.21 \\
\hline 25. & 2010-24A x RSV 1996 & -6.51 & $-22.38 * *$ & $-11.94 *$ & $-14.65 *$ & $-18.33 *$ & -11.93 & $24.11 *$ & 6.07 & 12.92 \\
\hline 26. & 2010-24A x RSV 1850 & -0.79 & $-14.83 * *$ & $-10.99 *$ & -6.70 & -10.72 & -3.73 & -4.05 & -15.84 & -15.75 \\
\hline 27. & 2010-24A x RSV 1837 & 6.14 & -11.49 & -0.69 & -9.93 & $-15.19 *$ & -5.37 & 15.26 & 1.92 & 0.15 \\
\hline 28. & 2010-24A x RSV 2124 & -5.78 & $-23.28 * *$ & -8.55 & 9.38 & 2.80 & $15.16 * *$ & 18.48 & 4.40 & 3.41 \\
\hline 29. & 2010-24A x PSR 34 & 4.63 & $-12.56 * *$ & -2.41 & $-13.23 *$ & -14.28 & -13.43 & 13.38 & 2.48 & -4.21 \\
\hline 30. & 2010-24A x CSV 26 & -3.43 & $-22.00 * *$ & -5.03 & $-20.61 * *$ & $-23.72 * *$ & $-18.44 *$ & 5.92 & -4.06 & -10.74 \\
\hline 31. & CMS 185A x RSV 2015 & 2.23 & $-13.07 * *$ & -3.94 & $-12.36 *$ & $-27.85 * *$ & -3.20 & 11.09 & -7.03 & 14.15 \\
\hline 32. & CMS 185A x RSV 2121 & -8.02 & $-24.11 * *$ & -9.61 & 3.85 & $-13.89 *$ & 13.42 & 19.11 & 2.80 & 17.13 \\
\hline 33. & CMS 185A x RSV 2138 & 0.60 & $-17.65 * *$ & 0.06 & -9.78 & $-27.57 * *$ & 3.70 & 11.79 & -2.48 & 8.35 \\
\hline 34. & CMS 185A x RS 585 & -1.96 & $-14.14 * *$ & $-11.53 *$ & -8.91 & $-18.94 * *$ & -9.86 & 5.44 & -6.13 & 0.51 \\
\hline 35. & CMS 185A x RSV 1996 & -6.27 & $-21.15 * *$ & $-10.55^{*}$ & $-16.95 * *$ & $-25.09 * *$ & $-19.22 *$ & -18.22 & $-27.33 *$ & -22.64 \\
\hline 36. & CMS 185A x RSV 1850 & $-9.56 *$ & $-21.28 * *$ & $-17.72 * *$ & 10.84 & -0.01 & 7.82 & 19.47 & 9.10 & 9.22 \\
\hline 37. & CMS 185A x RSV 1837 & -3.21 & $-18.21 * *$ & -8.22 & 4.42 & -7.21 & 3.54 & -10.30 & -17.39 & -18.83 \\
\hline 38. & CMS 185A x RSV 2124 & -7.82 & $-23.97 * *$ & -9.38 & $19.32 * *$ & 5.84 & $18.58^{*}$ & $36.13 * *$ & $24.91 *$ & $23.73 *$ \\
\hline 39. & CMS 185A x PSR 34 & -1.71 & $-16.76 * *$ & -7.10 & -10.97 & $-17.26 *$ & $-16.45^{*}$ & -5.77 & -11.18 & -16.98 \\
\hline 40 & CMS 185A x CSV 26 & -1.79 & $-19.76 * *$ & -2.19 & 2.61 & -7.09 & 0.65 & -7.68 & -12.80 & -18.87 \\
\hline & S.E. $($ Sij $) \pm$ & 9.18 & 10.61 & 10.61 & 94.42 & 114.80 & 114.80 & 9.16 & 10.57 & 10.57 \\
\hline
\end{tabular}

Note: * Significant at $5 \%$ level of significance, ${ }^{* *}$ Significant at $1 \%$ level of significance 
Contid...

\begin{tabular}{|c|c|c|c|c|c|c|c|c|c|c|}
\hline \multirow{3}{*}{$\begin{array}{l}\text { Sr. } \\
\text { No. }\end{array}$} & \multirow[t]{3}{*}{ Crosses } & \multicolumn{3}{|c|}{1000 grain weight $(\mathrm{g})$} & \multicolumn{3}{|c|}{ Dry fodder weight (g) } & \multicolumn{3}{|c|}{ Dry matter content (g) } \\
\hline & & \multicolumn{3}{|c|}{7} & \multicolumn{3}{|c|}{8} & \multicolumn{3}{|c|}{9} \\
\hline & & $\mathrm{MP}(\mathrm{H} 1)$ & $\mathrm{BP}(\mathrm{H} 2)$ & $\begin{array}{c}\text { Check } \\
\text { (H3) }\end{array}$ & MP (H1) & $\mathrm{BP}(\mathrm{H} 2)$ & $\begin{array}{c}\text { Check } \\
\text { (H3) }\end{array}$ & $\mathrm{MP}(\mathrm{H} 1)$ & $\mathrm{BP}(\mathrm{H} 2)$ & $\begin{array}{c}\text { Check } \\
\text { (H3) }\end{array}$ \\
\hline 1. & 2010-10A x RSV 2015 & $19.55^{* *}$ & 2.88 & $20.22 * *$ & $-20.54 *$ & $-30.54 * *$ & -15.10 & -9.25 & $-17.33 * *$ & -3.54 \\
\hline 2. & 2010-10A x RSV 2121 & -5.03 & $-18.27 * *$ & -4.49 & $-20.72 * *$ & $-33.42 * *$ & -10.41 & $-13.46^{*}$ & $-25.58 * *$ & -0.84 \\
\hline 3. & 2010-10A x RSV 2138 & 0.0 & $-17.39 * *$ & 6.74 & 6.88 & -6.65 & 14.33 & -8.60 & $-20.68 * *$ & 3.41 \\
\hline 4. & 2010-10A x RSV 585 & $11.66 * *$ & 3.41 & 2.25 & 0.97 & -9.37 & -0.17 & -7.07 & -11.11 & -6.64 \\
\hline 5. & 2010-10A x RSV 1996 & 6.43 & -5.21 & 2.25 & 0.70 & -9.70 & 4.10 & $-13.89 *$ & $-16.04 *$ & -15.25 \\
\hline 6. & 2010-10A x RSV 1850 & $23.68 * *$ & $22.08 * *$ & 5.62 & -14.54 & $-24.48 * *$ & -9.98 & $-14.49 *$ & $-21.83 * *$ & -9.48 \\
\hline 7. & 2010-10A x RSV 1837 & $24.32 * *$ & 4.55 & $29.21 * *$ & 15.59 & 3.50 & 19.71 & 1.74 & -5.72 & 5.96 \\
\hline 8. & 2010-10A x RSV 2124 & 3.03 & -5.56 & -4.49 & $-22.14 * *$ & $-31.62 * *$ & -17.32 & $-14.64 *$ & $-19.75 * *$ & -12.56 \\
\hline 9. & 2010-10A x PSR 34 & $14.29 * *$ & $11.39 *$ & -1.12 & 0.98 & -10.35 & 5.72 & -8.36 & -10.40 & -10.05 \\
\hline 10. & 2010-10A x CSV 26 & 6.01 & $-10.19 * *$ & 8.99 & -4.92 & -19.38 & 5.97 & 5.74 & 0.17 & 7.39 \\
\hline 11. & 2010-16A x RSV 2015 & $21.35 * *$ & 3.85 & $21.35 * *$ & 7.67 & -9.80 & 10.24 & -12.77 & $-22.16 * *$ & -9.17 \\
\hline 12. & 2010-16A x RSV 2121 & -1.12 & $-15.38 * *$ & -1.12 & 5.88 & -14.58 & 14.93 & -15.50 & $-28.72 * *$ & -5.03 \\
\hline 13. & 2010-16A x RSV 2015 & 2.65 & $-15.65 * *$ & 8.99 & -2.87 & -18.70 & -0.43 & $-18.91 * *$ & $-30.97 * *$ & -10.01 \\
\hline 14. & 2010-16A x RSV 2138 & 3.70 & -4.55 & -5.62 & 7.42 & -6.04 & 3.50 & 0.27 & -6.15 & -1.44 \\
\hline 15. & 2010-16A x RS 585 & 2.35 & $-9.38 *$ & -2.25 & 11.37 & -4.44 & 10.15 & -7.85 & -12.13 & -11.31 \\
\hline 16. & 2010-16A x RSV 1996 & $49.67 * *$ & $46.75^{* *}$ & $26.97 * *$ & -10.13 & $-23.94 * *$ & -9.34 & $-16.05 * *$ & $-24.84 * *$ & -12.96 \\
\hline 17. & 2010-16A x RSV 1837 & 6.52 & $-10.91 * *$ & $10.11 *$ & 7.36 & -8.00 & 6.40 & 0.39 & -8.91 & 2.37 \\
\hline 18. & 2010-16A x RSV 2124 & $25.61 * *$ & $14.44 * *$ & $15.73 * *$ & $23.04 * *$ & 3.53 & $25.17 *$ & $26.81 * *$ & $16.69 * *$ & $27.14 * *$ \\
\hline 19. & 2010-16A x PSR 34 & $20.26 * *$ & $16.46^{* *}$ & 3.37 & 17.22 & -0.36 & 17.49 & 6.81 & 2.11 & 2.51 \\
\hline 20. & 2010-16A x CSV 26 & -2.20 & $-17.59 * *$ & 0.00 & $44.10 * *$ & $17.30 *$ & $54.18 * *$ & $15.46^{*}$ & 7.03 & $14.75 *$ \\
\hline
\end{tabular}


Contid...

\begin{tabular}{|c|c|c|c|c|c|c|c|c|c|c|}
\hline \multirow[t]{3}{*}{ Sr. No. } & \multirow[t]{3}{*}{ Crosses } & \multicolumn{3}{|c|}{1000 grain weight $(\mathrm{g})$} & \multicolumn{3}{|c|}{ Dry fodder weight (g) } & \multicolumn{3}{|c|}{ Dry matter content (g) } \\
\hline & & \multicolumn{3}{|c|}{7} & \multicolumn{3}{|c|}{8} & \multicolumn{3}{|c|}{9} \\
\hline & & MP (H1) & $\mathrm{BP}(\mathrm{H} 2)$ & $\begin{array}{c}\text { Check } \\
\text { (H3) }\end{array}$ & MP (H1) & $\mathrm{BP}(\mathrm{H} 2)$ & $\begin{array}{c}\text { Check } \\
\text { (H3) }\end{array}$ & MP (H1) & $\mathrm{BP}(\mathrm{H} 2)$ & $\begin{array}{l}\text { Check } \\
\text { (H3) }\end{array}$ \\
\hline 21. & 2010-24A x RSV 2015 & $16.67 * *$ & 0.96 & $17.98 * *$ & -13.62 & $-22.86 * *$ & -5.72 & -2.35 & $-15.18 *$ & -1.02 \\
\hline 22. & 2010-24A x RSV 2121 & $-10.00^{*}$ & $-22.12 * *$ & -8.99 & $-20.31 * *$ & $-31.71 * *$ & -8.11 & $-12.51 *$ & $-28.00 * *$ & -4.07 \\
\hline 23. & 2010-24A x RSV 2138 & -5.76 & $-21.74 * *$ & 1.12 & $-17.94 *$ & $-26.79 * *$ & -10.32 & -5.13 & $-21.26 * *$ & 2.66 \\
\hline 24. & 2010-24A x RS 585 & 3.66 & -3.41 & -4.49 & -3.43 & -9.60 & -0.43 & 6.03 & -3.55 & 1.29 \\
\hline 25. & 2010-24A x RSV 1996 & $-9.30 *$ & $-18.75 * *$ & $-12.36 * *$ & -26.77 & $-32.86 * *$ & -22.61 & -6.24 & -13.16 & -12.35 \\
\hline 26. & 2010-24A x RSV 1850 & $18.95 * *$ & $18.18 * *$ & 2.25 & -26.99 & $-34.07 * *$ & -21.42 & $-17.45^{* *}$ & $-28.06 * *$ & $-16.69 *$ \\
\hline 27. & 2010-24A x RSV 1837 & $-9.68 *$ & $-23.64 * *$ & -5.62 & -8.44 & -16.19 & -3.07 & -4.45 & $-15.65 *$ & -5.20 \\
\hline 28. & 2010-24A x RSV 2124 & 7.23 & -1.11 & 0.00 & -2.40 & -12.42 & 5.89 & 3.54 & -7.35 & 0.95 \\
\hline 29. & 2010-24A x PSR 34 & 7.10 & 5.06 & -6.74 & $-16.63 *$ & $-24.35 * *$ & -10.79 & -5.24 & -12.01 & -11.67 \\
\hline 30. & 2010-24A x CSV 26 & -4.35 & $-18.52 * *$ & -1.12 & $-25.60 * *$ & $-35.61 * *$ & -15.36 & $-13.74 *$ & $-22.26 * *$ & $-16.65^{*}$ \\
\hline 31. & $185 A \times$ RSV 2015 & $20.43 * *$ & 7.69 & $25.84 * *$ & $-30.93 * *$ & $-40.38 * *$ & $-27.13 * *$ & $-23.26 * *$ & $-29.81 * *$ & $-18.09 * *$ \\
\hline 32. & $185 \mathrm{~A}$ x RSV 2121 & $20.43 * *$ & 7.69 & $25.84 * *$ & -9.68 & $-25.05 * *$ & 0.85 & $-11.95^{*}$ & $-24.00 * *$ & 1.26 \\
\hline 33. & $185 \mathrm{~A}$ x RSV 2138 & 3.55 & $-11.30 * *$ & $14.61 * *$ & -0.57 & -14.25 & 5.03 & $-13.18 *$ & $-24.37 * *$ & -1.40 \\
\hline 34. & $185 \mathrm{~A} \times \mathrm{RS} 585$ & $17.65^{* *}$ & $13.64 * *$ & $12.36^{* *}$ & -4.01 & -13.32 & -4.52 & -0.85 & -4.75 & 0.04 \\
\hline 35. & 185A x RSV 1996 & $8.99 *$ & 1.04 & 8.99 & -4.50 & -15.47 & -2.56 & -2.29 & -4.30 & -3.41 \\
\hline 36. & 185A x RSV 1850 & $25.79 * *$ & $21.95 * *$ & $12.36^{* *}$ & -0.64 & -13.31 & 3.33 & -1.88 & -9.95 & 4.29 \\
\hline 37. & 185A x RSV 1837 & 6.25 & -7.27 & $14.61 * *$ & -4.59 & -15.68 & -2.47 & $-13.44 *$ & $-19.45 * *$ & -9.48 \\
\hline 38. & 185A x RSV 2124 & $11.63 * *$ & 6.67 & 7.87 & -11.13 & $-22.94 * *$ & -6.83 & 0.74 & -4.89 & 3.63 \\
\hline 39. & $185 \mathrm{~A}$ x PSR 34 & 3.11 & 1.22 & -6.74 & -3.78 & -15.67 & -0.55 & -4.35 & -6.08 & -5.71 \\
\hline 40 & $185 \mathrm{~A} \times \mathrm{CSV} 26$ & $-12.63 * *$ & $-23.15 * *$ & -6.74 & -13.95 & $-27.91 * *$ & -5.25 & -6.14 & -10.71 & -4.27 \\
\hline & S.E. $(\mathbf{S i j}) \pm$ & 1.18 & 1.36 & 1.36 & 6.80 & 7.85 & 7.85 & 10.88 & 12.57 & 12.57 \\
\hline
\end{tabular}

Note: * Significant at $5 \%$ level of significance, $* *$ Significant at $1 \%$ level of significance 
Contid...

\begin{tabular}{|c|c|c|c|c|c|c|c|}
\hline \multirow{3}{*}{$\begin{array}{l}\text { Sr. } \\
\text { No. }\end{array}$} & \multirow[t]{3}{*}{ Crosses } & \multicolumn{3}{|c|}{ Grain yield per plant (g) } & \multicolumn{3}{|c|}{ Harvest index (\%) } \\
\hline & & \multicolumn{3}{|c|}{10} & \multicolumn{3}{|c|}{11} \\
\hline & & $\mathrm{MP}(\mathrm{H} 1)$ & $\mathrm{BP}(\mathrm{H} 2)$ & Check (H3) & MP (H1) & $\mathrm{BP}(\mathrm{H} 2)$ & $\begin{array}{c}\text { Check } \\
\text { (H3) }\end{array}$ \\
\hline 1. & 2010-10A x RSV 2015 & -2.07 & $-14.11 *$ & 8.49 & 8.76 & 4.18 & $13.69 *$ \\
\hline 2. & 2010-10A x RSV 2121 & 4.12 & -8.87 & 15.66 & $16.67 * *$ & $16.03 *$ & $17.24 * *$ \\
\hline 3. & 2010-10A x RSV 2138 & 0.07 & $-14.58 *$ & 14.67 & 9.68 & 7.56 & 11.81 \\
\hline 4. & 2010-10A x RSV 585 & -12.00 & $-17.00^{*}$ & -10.82 & -3.78 & -5.46 & -2.12 \\
\hline 5. & 2010-10A x RSV 1996 & -1.48 & -4.88 & -2.69 & $13.61 *$ & 12.53 & $14.62 *$ \\
\hline 6. & 2010-10A x RSV 1850 & -8.55 & -14.02 & -6.99 & 6.56 & 3.08 & 3.01 \\
\hline 7. & 2010-10A x RSV 1837 & 8.16 & 0.99 & 10.89 & 8.51 & 6.93 & 6.85 \\
\hline 8. & 2010-10A x RSV 2124 & $21.93 * *$ & $-25.34 * *$ & $-22.08 * *$ & -7.18 & -8.89 & -8.96 \\
\hline 9. & 2010-10A x PSR 34 & 6.40 & 11.16 & -2.83 & $17.54 * *$ & 10.38 & 10.30 \\
\hline 10. & 2010-10A x CSV 26 & 15.55 & 13.01 & 12.59 & 8.66 & 5.15 & 5.07 \\
\hline 11. & 2010-16A x RSV 2015 & -23.40 & $-34.16 * *$ & -16.84 & $-11.83^{*}$ & $-15.32 *$ & -7.59 \\
\hline 12. & 2010-16A x RSV 2121 & -13.23 & $-25.56 * *$ & -5.53 & -0.77 & -1.04 & -0.01 \\
\hline 13. & 2010-16A x RSV 2015 & -11.76 & $-26.03 * *$ & -0.69 & 4.16 & 2.42 & 6.48 \\
\hline 14. & 2010-16A x RSV 2138 & -3.32 & -10.80 & -4.15 & -0.18 & -1.65 & 1.82 \\
\hline 15. & 2010-16A x RS 585 & -9.55 & -14.62 & -12.66 & -1.41 & -2.07 & -0.25 \\
\hline 16. & 2010-16A x RSV 1996 & -4.58 & -12.23 & -5.05 & $13.57 *$ & 9.57 & 10.11 \\
\hline 17. & 2010-16A x RSV 1837 & 6.37 & -2.81 & 6.71 & 6.89 & 5.04 & 5.55 \\
\hline 18. & 2010-16A x RSV 2124 & $38.89 * *$ & $29.88 * *$ & $35.56 * *$ & 10.97 & 8.63 & 9.16 \\
\hline 19. & 2010-16A x PSR 34 & 8.35 & 6.31 & -3.44 & 1.45 & -4.98 & -4.51 \\
\hline 20. & 2010-16A x CSV 26 & 2.38 & -2.14 & -2.50 & $-11.89 *$ & $-14.97 *$ & $-14.55^{*}$ \\
\hline 21. & 2010-24A x RSV 2015 & 10.91 & -7.67 & 16.62 & $13.91 *$ & 8.22 & $18.10^{* *}$ \\
\hline 22. & 2010-24A x RSV 2121 & -14.25 & $-28.75^{* *}$ & -9.58 & -4.66 & -5.98 & -5.00 \\
\hline
\end{tabular}




\begin{tabular}{|c|c|c|c|c|c|c|c|}
\hline 23. & 2010-24A x RSV 2138 & -1.18 & $-19.68 * *$ & 7.83 & 5.65 & 2.75 & 6.81 \\
\hline 24. & 2010-24A x RS 585 & 9.48 & -2.47 & 4.80 & 2.93 & 0.30 & 3.84 \\
\hline 25. & 2010-24A x RSV 1996 & -6.37 & -14.75 & -12.80 & 0.71 & -1.08 & 0.76 \\
\hline 26. & 2010-24A x RSV 1850 & -6.87 & $-17.28 * *$ & -10.51 & 11.40 & 8.67 & 6.75 \\
\hline 27. & 2010-24A x RSV 1837 & -0.17 & -11.90 & -3.27 & 5.98 & 5.32 & 3.47 \\
\hline 28. & 2010-24A x RSV 2124 & $24.64 * *$ & 12.47 & 17.39 & $20.19 * *$ & $18.97 * *$ & $16.88 *$ \\
\hline 29. & 2010-24A x PSR 34 & 3.39 & 1.36 & -11.40 & 8.93 & 3.13 & 1.31 \\
\hline 30. & 2010-24A x CSV 26 & -7.67 & -14.92 & -15.23 & 7.75 & 5.13 & 3.27 \\
\hline 31. & CMS 185A x RSV 2015 & -14.27 & $-31.66 * *$ & -13.68 & $12.95^{*}$ & -2.51 & 6.39 \\
\hline 32. & CMS 185A x RSV 2121 & 4.25 & $-17.04 *$ & 5.28 & $16.50 *$ & 3.94 & 5.03 \\
\hline 33. & CMS $185 A$ x RSV 2138 & 3.76 & $-19.11 * *$ & 8.59 & $21.30 * *$ & 6.89 & 11.12 \\
\hline 34. & CMS 185A x RS 585 & -0.96 & -15.88 & -9.62 & -1.02 & $-12.62 *$ & -9.54 \\
\hline 35. & CMS 185A x RSV 1996 & -3.01 & -15.92 & -13.99 & -0.37 & -11.42 & -9.78 \\
\hline 36. & CMS 185A x RSV 1850 & $20.73^{*}$ & 2.25 & 10.62 & $25.73 * *$ & $16.20 *$ & 8.55 \\
\hline 37. & CMS 185A x RSV 1837 & 4.42 & -12.10 & -3.49 & $21.99 * *$ & 10.83 & 7.52 \\
\hline 38. & CMS 185A x RSV 2124 & $27.27 * *$ & 9.40 & 14.18 & $26.22 * *$ & $15.08 *$ & 10.76 \\
\hline 39. & CMS 185A x PSR 34 & -4.06 & -10.84 & $-22.07 * *$ & -0.27 & -5.10 & $-16.71 *$ \\
\hline \multirow[t]{2}{*}{40} & CMS 185A x CSV 26 & 4.55 & -8.34 & -8.68 & 8.72 & 0.46 & -6.11 \\
\hline & S.E. $($ Sij $) \pm$ & 3.95 & 4.57 & 4.57 & 1.57 & 1.81 & 1.81 \\
\hline
\end{tabular}

Note: * Significant at $5 \%$ level of significance, ** Significant at $1 \%$ level of significance 
Days to $50 \%$ flowering standard heterosis ranged from -5.61 to -4.08 per cent over check CSH-15R (Table 4). The cross CMS185A x RSV-2121 (-5.61\%) exhibits highest negative standard heterosis followed by cross RMS-2010-10A x RSV-1850 (-5.10), CMS185A x RSV-2015 (-5.00) and CMS-185A x RSV1996 (-5.00). Out forty hybrids, seven hybrids, were showed significant negative standard heterosis over the check CSH-15R. These results are in line with the earlier results of Prabhakar (2001), Kulkarni and Patil (2004), Umakant et al., (2006), Gite et al., (2015), Kalpande et al., (2015) and Iyanar and Gopalan (2016).

The magnitude of heterosis over mid parent was -3.54 per cent (CMS-185A x RS-585) to 6.46 per cent (CMS-185A x RSV-2015) for days to physiological maturity. The range of heterobeltiosis was from -3.94 per cent (RMS-2010-16A x RSV-2015) to -5.80 per cent (CMS-185A x RSV-2015). The standard heterosis ranged from 5.63 to -4.89 per cent. The crosses CMS-185A x RSV-2015 (4.89\%) and CMS-185A x RSV-2121 ($4.11 \%$ ) were showed highest negative standard heterosis. Similar results recorded by Prabhakar (2001), Umakant et al., (2006), Gite et al., (2015), Kalpande et al., (2015) and Prasad B et al., (2018).

Average heterosis for seedling height at 14 DAE ranged from -6.56 to 26.81 per cent (Table 4). The cross RMS-2010-10A x RSV1850 (26.81\%) exhibited highest positive average heterosis followed by RMS-201016A x RSV-1837 (20.43\%) and RMS-201010A x CSV-26 (17.86\%). Heterobeltiosis ranged from -7.94 per cent (CMS-185A x RSV-1850) to 23.82 per cent (RMS-201010A x RSV-1850) (Table 4). As the check $\mathrm{CSH}-15 \mathrm{R}$ is tall in growth habit the standard heterosis for Seedling height at $14 \mathrm{DAE}$ ranged from 7.21 to 30.13 per cent over check CSH-15R (Table 4). Twenty one crosses showed standard heterosis in desirable direction. Maximum positive heterosis was found in crosses RMS-2010-10A x RSV-1850 (30.13\%) followed by RMS-2010-10A x CSV-26 (19.94\%) and RMS-2010-16A $x$ RSV-1837 (19.66\%) while minimum positive standard heterosis was observed in CMS185A x RS-585 (7.21\%).

Out of forty hybrids, two hybrids recorded significant average heterosis in positive direction for plant height $(\mathrm{cm})$. The heterosis over better parent ranged from -25.41 per cent (RMS-2010-24A x RSV-2121) to - 11.97 per cent (RMS-2010-16A x RSV-1837). Out of forty crosses, none of the cross exhibited positive and significant heterobeltiosis for this trait.

The range of standard heterosis over the check CSH-15R was -17.72 per cent (CMS185A x RSV-1850) to 14.07 per cent (RMS2010-24A x RSV2138). Out of 40 crosses, three crosses exhibited positive and significant standard heterosis for this trait. Maximum positive heterosis was found in crosses RMS-2010-10A x RSV-2138 (14.07\%) followed by RMS-2010-10A x RSV-2015 (13.02\%) and RMS-2010-10A $x$ RSV-2121 (10.82\%). The similar results were earlier reported by Prabhakar (2001), Prakash et al., (2010), Gite et al., (2015), Kalpande et $a l .$, (2015) and Kumar and shrotria (2016).

Out of forty hybrids, two hybrids recorded significant average heterosis in positive direction for grains per panicle (no.). The heterosis over better parent ranged from 33.31 per cent (RMS-2010-24A x RSV-2121) to 26.05 per cent (RMS-2010-16A x RSV2124). Out of forty crosses, only one of the cross exhibited positive and significant heterobeltiosis for this trait.

The range of standard heterosis over the check CSH-15R was -19.22 per cent (CMS- 
$185 \times$ RSV-1996) to 41.22 per cent (RMS2010-216A x RSV2124). Out of forty crosses, four crosses exhibited positive and significant standard heterosis for this trait. Maximum positive heterosis was found in crosses RMS2010-216A x RSV-2124 (41.22\%) followed by RMS-2010-24A x RSV-2015 (23.64\%) and CMS-185A x RSV-2124 (18.58\%). Similar finding was recorded by Laxman (2001).

Average heterosis for panicle weight ranged from -33.46 to 36.13 per cent. The cross CMS185A x RSV-2124 (36.13\%) exhibited highest positive average heterosis followed by RMS-2010- 24A x RSV-1996 (24.11\%) and RMS-2010-24A x RSV-2015 (22.78\%). Heterobeltiosis ranged from -41.67 per cent (RMS-2010-16A x RS-585) to 24.91 per cent (CMS-185A x RSV-2124) (Table 4). Out of forty crosses, only one cross show highest significant positive heterobeltiosis in desirable direction for this trait. Standard heterosis over check hybrid CSH-15R ranged from 23.73 per cent (CMS-185A x RSV-2124 to 36.39 per cent (RMS-2010-16A x RSV2124). Three crosses showed standard heterosis in desirable direction. The highest significant standard heterosis recorded in the cross, RMS-2010-16A x RSV-2124 (36.39\%), followed by cross RMS-2010-10A $x$ RSV2015 (23.80\%) and CMS-185A x RSV$2124(23.73 \%)$ (Table 4). Similar findings reported by Gite et al., (2015), Prasad B et al., (2018) and Khadi et al., (2018).

The magnitude of heterosis over mid parent was 11.63 per cent (CMS-185A x RSV-2124) to 49.67 per cent (RMS-2010-16A x RSV1996) for 1000 grain weight $(\mathrm{g})$. The cross RMS-2010-16A x RSV-1996 (49.67\%) 71 exhibited highest positive average heterosis followed by CMS-185A x RSV-1850 (25.79\%) and RMS-2010-10A x RSV-1837 $(24.32 \%)$. The range of heterobeltiosis was from -23.64 per cent (RMS-2010-24A x RSV-
1837 ) to 46.75 per cent (RMS-2010-16A $x$ RSV-1996). Out of forty crosses, seven crosses show highest significant positive heterobeltiosis in desirable direction for this traits. The standard heterosis ranged from 12.36 per cent to 29.21 per cent. The RMS2010-24A x RSV-1837 (29.21\%), RMS2010-16A x RSV-1996 (26.97\%), and CMS185A x RSV-2015 (25.84\%) were showed highest positive standard heterosis. Out of forty crosses, thirteen crosses show highest significant positive standard heterosis in desirable direction for this trait. Similar result recorded by Prabhakar (2001), Umakant et al., (2006), Gite et al., (2015) and Kalpande et al., (2015),

Out of forty hybrids, two hybrids recorded significant average heterosis in positive direction for dry fodder weight $(\mathrm{g})$. The heterosis over better parent ranged from 40.38 per cent (CMS-185A x RSV-2015) to 17.30 per cent (RMS-2010-216A x CSV-26). Out of forty crosses, only one of the cross exhibited positive and significant heterobeltiosis for this trait. The range of standard heterosis over the check CSH-15R was -27.13 per cent (CMS-185 x RSV-2015) to 54.18 per cent (RMS-2010-16A x CSV26).

Out of forty crosses, two crosses exhibited positive and significant standard heterosis for this trait. Maximum positive heterosis was found in crosses RMS-2010-216A x CSV-26 $(54.18 \%)$ followed by RMS-2010-16A x RSV-2124 (25.17\%) respectively. Similar result reported by Prakash et al., (2010) and Kumar and shrotria (2016).

Average heterosis for dry matter content ranged from -23.26 to 26.81 per cent. The cross RMS-2010-16A x RSV-2124 (26.81\%) exhibited highest positive average heterosis followed by RMS-2010-16A x CSV-26 $(15.46 \%)$. Heterobeltiosis ranged from -30.97 
(RMS-2010-16A x RSV2015) to 16.69 per cent (RMS-2010-16A x RSV-2124) (Table 4). Out of forty crosses, only one cross show highest significant positive heterobeltiosis in desirable direction for this trait. Standard heterosis over check hybrid $\mathrm{CSH}-15 \mathrm{R}$ ranged from -18.09 per cent (CMS-185A x RSV$2015)$ to 27.14 per cent (RMS-2010-16A $\mathrm{x}$ RSV-2124). Two crosses showed standard heterosis in desirable direction. The highest significant standard heterosis recorded in the cross, RMS-2010-16A x RSV2124 (27.14\%), followed by cross RMS-2010-16A x CSV-26 (14.75\%), (Table 4). Similar finding was reported by Akabari et al., (2012).

The magnitude of heterosis over mid-parent for grain yield per plant ranged from 21.93 per cent (RMS-2010-10A x RSV-2124) to 38.89 per cent (RMS-2010-16A x RSV2124). A total of four hybrids recorded significant positive heterosis over mid parent. The cross RMS-2010-16A x RSV2124 (38.89\%) exhibited highest significant average heterosis followed by CMS-185 x RSV-2015 72 (27.27\%) and RMS-2010-24A x RSV-2124 (24.64\%) (Table 4). The range in heterobeltiosis varied from -31.66 per cent (CMS-185 x RSV-2015) to 29.88 per cent (RMS-2010-16A x RSV-2124). A only one hybrid recorded significant positive heterosis over better parent. The cross RMS-2010-16A x RSV-2124 (29.88\%) exhibited highest significant heterosis over better parent. The range of heterosis over standard checks, $\mathrm{CSH}-$ 15R was from -22.08 per cent (RMS-2010$10 \mathrm{~A} \times \mathrm{RSV}-2124)$ to 35.56 per cent (RMS2010-16A x RSV-2124).

Among forty hybrids, only one hybrid recorded positive significant heterosis over CSH-15R. The highest standard heterosis in desirable direction was recorded in the cross RMS-2010-16A x RSV-2124 (35.56\%), (Table 4). The similar results were earlier reported by Prabhakar (2001), Kulkarni and Patil (2004), Umakant et al., (2006). Gite et al., (2015), Kalpande et al., (2015), Khadi et al., (2018) and Prasad et al., (2018).

A total of twelve hybrids recorded significant positive heterosis over mid parent for Harvest index $(\%)$. The magnitude of heterosis over mid-parent for harvest index ranged from 11.89 per cent (RMS-2010- 16A x CSV-26) to 26.22 per cent (CMS-185A x RSV-2124). The heterosis over better parent ranged from 15.32 per cent (RMS-2010-16A x RSV-2015) to 18.97 per cent (RMS-2010-24A x RSV2124). Out of forty crosses, four crosses exhibited positive and significant heterobeltiosis for this trait. The range of standard heterosis over the check CSH-15R was -16.71 per cent (CMS-185 x RSV-PSR$34)$ to 18.10 per cent (RMS-2010-24A $x$ RSV-2015). Out of forty crosses, five crosses exhibited positive and significant standard heterosis for this trait. Maximum positive heterosis was found in crosses RMS-201024A x RSV-2015 (18.10\%) followed by RMS-2010-10A x RSV-2121 (17.24\%) and RMS-2010-24A X RSV-2124 (16.88\%) respectively. Similar finding was reported by Jadhav and Deshmukh (2017).

In conclusions the heterosis studies indicated that expression of relative heterosis, heterobeltosis and standard heterosis in several crosses for most of the characters in both desirable direction as well as undesirable direction. Four crosses were shown significant positive heterosis over standard check CSH-15R for the traits days to $50 \%$ flowering, days to physiological maturity, grains per panicle, panicle weight, dry fodder weight.

The cross RMS-2010-16A x RSV-2124 show significant standard heterosis over check $\mathrm{CSH}-15 \mathrm{R}$ for grain yield per plant. Thus there is need of cytoplasm diversification and use of diverse parents in rabi sorghum for hybrids development. 


\section{Acknowledgement}

The Authors are sincerely grateful to Department of Agriculture Botany and All India Coordinated Sorghum Improvement Project, Mahatma Phule Krushi Vidyapeeth Rahuri, for providing necessary facilities for complete research work.

\section{References}

Akabari, V.R., Parmar, H.P., Niranjana, M. and Nakarani, D.B. (2012). Heterosis and combining ability for green fodder yield and its contributing traits in forage sorghum [Sorghum bicolor (1.) moench]. Forage Res., 38(3): 156-163.

Dillon, S.L., Shapter, F.M., Henry, R.J., Cordeiro, G., Izquierdo, L. and Slade, L.L. 2007. Domestication to crop improvement: genetic resources for sorghum and saccharum (Andropogoneae). Ann. Bot. (Lond.) 100: 975-989.

Fonseca, A. and Patterson. F.L. 1968. Hybrid vigour in a seven parent diallel cross in common winter wheat ( $T$. aestivum L.). Crop Sci., 8: 85-88.

Gite, A.G., Kute, N.S. and Patil, V.R. (2015). Heterosis studies for yield and its components traits rabi sorghum (Sorghum bicolor L. Moench). J. of Global Biosci., 4(8): 3207-3219.

Iyanar, K. and Gopalan, A. (2006). Heterosis In relation to per se and sca effects in grain sorghum \{Sorghum bicolor (1.) moench\}. Indian J. Agric. Res., 40(2): 109-113.

Jadhav, R.R. and Deshmukh, D.T. (2017). Heterosis and combining ability studies in sorghum (Sorghum bicolor (L.) Moench) over the environments. Int. J. Curr. Microbiol. Ap. Sci., 6(10): 3058-3064.

Kalpande, V.V., Ghorade, R.B., Nair, B., Kahate, N.S. and Gunjal, S.M. (2015) Heterosis studies for grain yield and yield components in post rainy sorghum. Plant Archives., 15(1): 177-180.

Khadi, P.S., Biradar, B.D. and Pattanashetti, S.K. (2018). Heterosis studies for yield and yield components in rabi sorghum [Sorghum bicolor (L.) Moench]. J. Farm Sci., 31(3): 342-343.

Kulkarni, V. and Patil, M.S. (2004). Heterosis Studies in Sorghum. Karnataka J. Agri. Sci., 17(3): 458-459.

Kumar, P. and Shrotria, P.K. (2016). Combining ability and heterosis studies for yield and component traits in forage sorghum (Sorghum bicolor L. Moench). Green Farming., 7(1):1-7.

Laxman., S. (2001). Studies on heterosis and combining ability in sorghum (Sorghum bicolor L. Moench) through Line x Tester Analysis. J. Res. Angrau., 29(4): 12-17.

Prabhakar (2001). Heterosis in rabi sorghum (Sorghum bicolor (L.) Moench). Indian J. Genet., 61(4): 364-365.

Prakash, R., Ganesamurthy, K., Nirmalakumari, A. and Nagarajan, P. (2010). Heterosis for fodder yield in sorghum (Sorghum bicolor L. Moench). Electronic J. of Plant Breed., 1(3): 319-327.

Prasad B, H. V., Biradar, B.D. and Verma, L.K. (2018). Estimation of heterosis among $B \mathrm{X}$ $\mathrm{B}, \mathrm{B} \times \mathrm{R}$ and $\mathrm{R} \times \mathrm{R}$ crosses of rabi sorghum. Bull. Env. Pharmacol. Life Sci., 7(1): 14-20.

Purseglove, J.W. 1972. Sorghum bicolor (L.) Monech, in Tropical crops. Monocotyledons, longman group limited, London, pp. 261-287.

Umakanth, A.V., Rao, S.S. and Kuriakose, S.V. (2006). Heterosis in landrace hybrids of post-rainy sorghum $\{$ Sorghum bicolor (1.) moench\}. Indian J. Agric. Res., 40 (2): 147-150.

\section{How to cite this article:}

Totre, A. S., A.S. Jadhav, M. S. Shinde, N. S. Kute, U. S. Dalvi, R. S. Bhoge and Shinde, G. C. 2020. Heterosis for Grain Yield and its Component Traits in Rabi Sorghum. Int.J.Curr.Microbiol.App.Sci. 9(11): 846-863. doi: https://doi.org/10.20546/ijcmas.2020.911.102 